\title{
NEUTRON FLUX DETERMINATION BY HIGH ACCURACY TEMPERATURE MEASUREMENT
}

\author{
J. Svoboda ${ }^{1, a, b}$ \\ a Joint Institute for Nuclear Research, Joliot-Curie 6, 141980 Dubna, Russian Federation \\ ${ }^{b}$ Brno University of Technology, Technicka 3058/10, 61600 Brno, Czech Republic \\ E-mail: svobodaljinr.ru
}

J. Adam ${ }^{\text {a,c }}$, A. Baldin a, S. Gustov ${ }^{\text {a }}$, K. Katovsky ${ }^{\text {b }}$, J. Khushvaktov ${ }^{\text {a }}$, I. Mar'in ${ }^{\text {, }}$, A. Solnyshkin a , P. Tichy ${ }^{\text {a,c,d }}$, S. Tyutyunnikov ${ }^{a}$, R. Vespalec ${ }^{a, d}$, M. Zeman ${ }^{\text {a,b }}$ ${ }^{c}$ Nuclear Physics Institute of the ASCR, v. v. i., Rez 130, 25068 Rez, Czech Republic

${ }^{d}$ Czech Technical University in Prague, V Holesovickach 2, 18000 Prague, Czech Republic

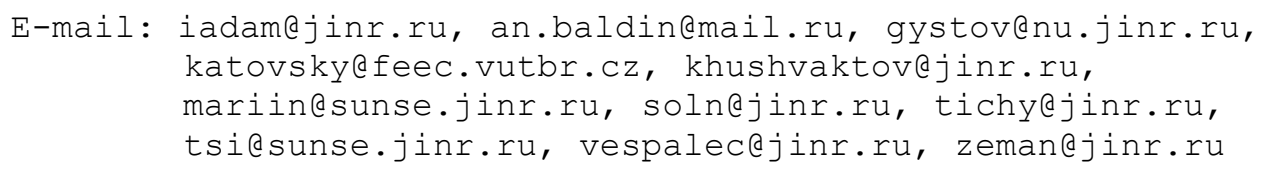

This paper is focused on the description of developing a method to determine neutron flux by high-accuracy temperature measurement. The paper deals with the procedure of sophisticated measurement, its analyzation and brings part of reached results. The method is in development in the research group aimed at ADS in the DLNP, JINR. Since the first experiment in November 2015, another 5 experiments have been performed and due to progress, future experiment seems to be closer to reach relatively cheap probe with the ability to determine the neutron flux online.

The 26th International Nuclear Physics Conference

11-16 September, 2016

Adelaide, Australia

${ }^{1}$ Speaker

This work was financially supported by Ministry of Youth and Education and Ministry of Finances of the Czech Republic. The project was also supported from the LO1210, FEKT-S-14-2520 projects at the BUT. 


\section{Introduction}

There is scientists group with long-term research aimed at the behavior of the spallation targets in the Dzhelepov Laboratory of Nuclear Problems which closely cooperate with a group from Veksler and Baldin Laboratory of High Energy Physics in the JINR. The main topics of the group's research are neutron production for heat generation and transmutation of the spent fuel at deep subcritical ADS. Group also hold an investigation of the core with maximally hard neutron spectrum. But there is also the parallel research aimed at the determination of the neutron flux by temperature since November 2015.

The main goal of this ongoing research is to determine neutron gain in all sections of QUINTA Target Assembly (TA). A parallel goal of the research is to use this method to determine the neutrons leakage. Until now, 6 experiments were performed in November and December 2015, March, May, June and December 2016, respectively. This article summarizes first five experiments. Thermocouples used as the probes measure the temperature of samples as ${ }^{232} \mathrm{Th}$, ${ }^{\text {nat }} \mathrm{U}$ and enriched ${ }^{235} \mathrm{U}$. Probes are inputted into narrow space about $17 \mathrm{~mm}$ located between two adjoining sections of QUINTA TA which has 5 section in total. Results from these experiments going to be compared with MCNPX simulation and activation foil techniques. Measured temperature consists of heat released by neutron fission mainly combined with heat transfer from the target cylinders. Reached temperature needs to be sophisticated recalculate to determine neutron flux, respectively neutron gain. The TA QUINTA is irradiated by proton beam with energy about $660 \mathrm{MeV}$ and generally, the temperature during 6 hours' experiment may rise to $34{ }^{\circ} \mathrm{C}$, so the temperature difference goes to $12{ }^{\circ} \mathrm{C}$.

\subsection{Spallation natural uranium target assembly QUINTA}

All experiments described in this article were set at spallation target QUINTA, see in Figure 1. QUINTA which consists of five hexagonal sections where the mass of natural uranium reaches $512 \mathrm{~kg}$. Each section [2] with the size of $350 \mathrm{~mm}$ high to $114 \mathrm{~mm}$ length is composed of natural uranium cylinders with diameter $36 \mathrm{~mm}$, length $104 \mathrm{~mm}$ and mass about $1.72 \mathrm{~kg}$. The first section consists of 54 cylinders with a total mass of uranium about $92 \mathrm{~kg}$. All other sections (from the second to the fifth) are identified with 61 cylinders and the total mass of uranium up to $105 \mathrm{~kg}$. All sections are covered by aluminum plate shield with a thickness of $5 \mathrm{~mm}$ so the total size of QUINTA is $700 \times 350 \times 350 \mathrm{~mm}^{3}$ with total mass $540 \mathrm{~kg}$ [1]. Between each two sections, there is $17 \mathrm{~mm}$ of the air gap. About $10 \mathrm{~cm}$ thick lead shielding has been provided surrounding the target assembly (TA) QUINTA [2].
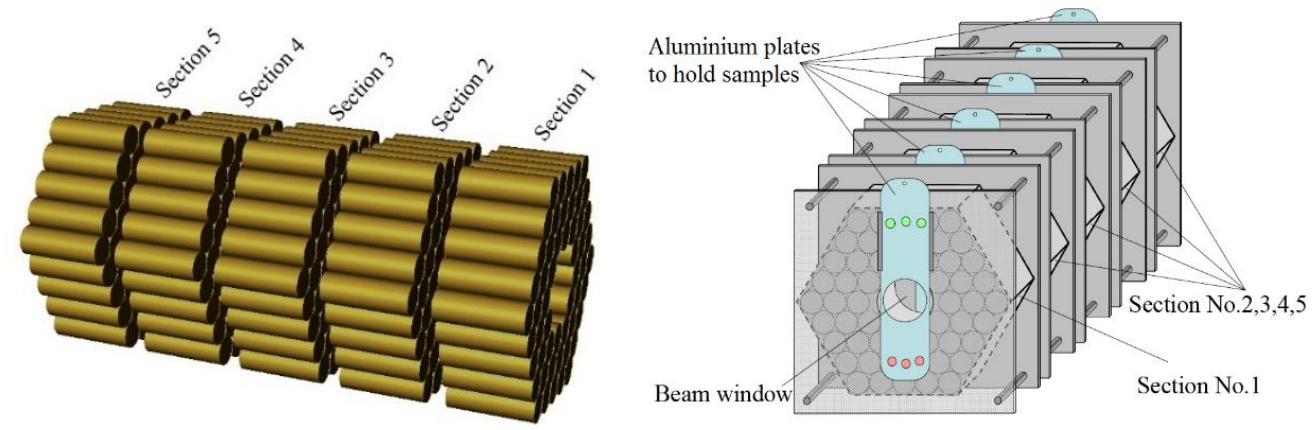

Figure 1: Uranium cylinders of TA QUINTA setup (left), all sections of TA QUINTA (right) [2]. 


\subsubsection{History and ongoing research in short}

Since 2009 this TA QUINTA has been used for a lot of research experiments at two accelerators in JINR. From 2009 till 2014 experiments were implemented at Nuclotron with proton energies up to $12.8 \mathrm{GeV}$ and intensity of $10^{9} \mathrm{~s}^{-1}$ [1]. Nuclotron had the ability to accelerate also deuterons (usually used for QUINTA experiments) and other various heavy ions. Since the middle of 2014 Nuclotron has been in the reconstruction. There is also new project NICA in construction with higher acceleration ability and $500 \mathrm{~m}$ in diameter.


Figure 2: Spallation target Buran (on the left) [2] planned to be installed in the first quarter of 2017 at the Phasotron irradiation facility (on the right side).

Due to this reconstruction, all research experiments have been moved to Phasotron since the middle of 2014. Phasotron can accelerate only protons to energy $660 \mathrm{MeV}$ with the intensity of $10^{13} \mathrm{~s}^{-1}$ (equal to current $1.6 \mu \mathrm{A}$ ). Next step of research activity of neutron flux in spallation target assemblies will be BURAN setup see Figure 2 (Russian abbreviation of "Large Uranium target") with diameter of $1.2 \mathrm{~m}$ and $1 \mathrm{~m}$ of length with mass of metallic depleted uranium about $20 \mathrm{t}[1]$.

\section{Neutron flux determination}

Determination of the neutron flux is more difficult in the narrow setting where larger detectors are not capable of working. To determine the neutron flux inside of the spallation target QUINTA, the activation analysis of various threshold foils is mostly used. A new method of the neutron flux determination has been developing as parallel monitor by the research group of J. Adam, DLNP, JINR. It is based on measurement of the temperature differences, online analyzing and the recalculation heat to a neutron flux. By interaction of neutrons with matter, the heat is released with dependence on material properties and neutron energies. That is the base of used principle for this method.

Only $17 \mathrm{~mm}$ air gap between each two sections is available to detect neutron flux. Measuring probe is set into this space and online monitoring by high accuracy thermocouples and precious analogue-digital (A/D) converter. Thermocouples type $\mathrm{T}$ from the OMEGA company are used (model TT-T-24-SLE-1000) because of its high voltage amplifying in the required range. The thermocouples are welded at the laboratory before each experiment and the weld is pressed to change the shape of the weld. It is done for the reason of better thermal contact with the measured material. This destruction of the thermocouple was tested and compared with normal sphere weld without destruction. No difference in accuracy was found by this test.

As the converters, the cards from National Instrument type NI 9214 and NI 9212 are used. Another problem is long distance measurement, caused by strong gamma radiation and neutron 
field which complicates the measurement. The distance between A/D converter and QUINTA is about $23 \mathrm{~m}$. Due to that, each experiment is more problematic to organize. After each experiment, the probes are measured by using of gamma-spectrometry to analyze and compare data from spectra to reached data from temperature measurement.
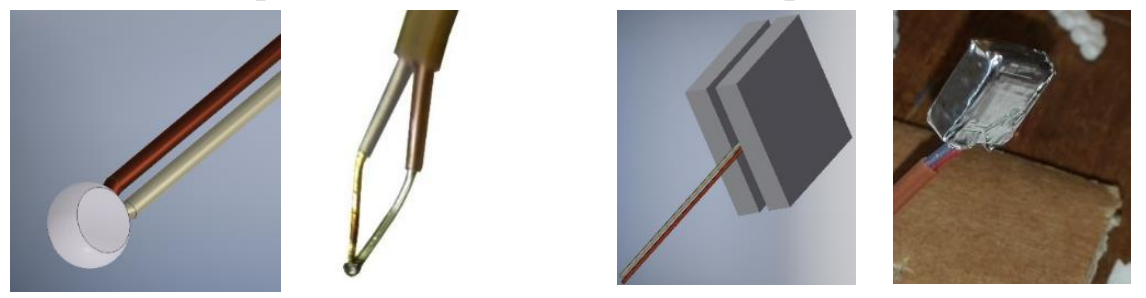

Figure 3: Model and the reality picture of measuring thermocouple (left) and probe (right).

Globally 6 experiments were performed. First one, so called pre-experiment, was aimed to determine temperature difference by standard TC behind the second section. The result ensures to choose the right one type of thermocouples and A/D converter for on-coming experiments. Since that experiment, the temperature of this setup was only calculated, never measured.

\subsection{Experiment - December 2015}

For the December experiment in 2015, 15 thermocouples (TC) were used. Thermocouples measured thermally insulated samples of ${ }^{235} \mathrm{U}$, nat $\mathrm{U}$, and ${ }^{232} \mathrm{Th}$. Nine TC were measured inside and $6 \mathrm{TC}$ outside. The thermocouples covered by measured sample were placed at the aluminum holder and putting into the narrow space between $2^{\text {nd }}$ and $3^{\text {rd }}$ section. The idea of this measurement was to monitor the heat distribution. The probe ${ }^{2}$ was thermally insulated by alumina foil to decrease thermal radiation heat exchange and next layer of special insulation foam to minimalize thermal conduction and convection. The idea was to measure heat released by fission inside of measured wafers. The samples mass is usually about $2 \mathrm{~g}$ per a probe.
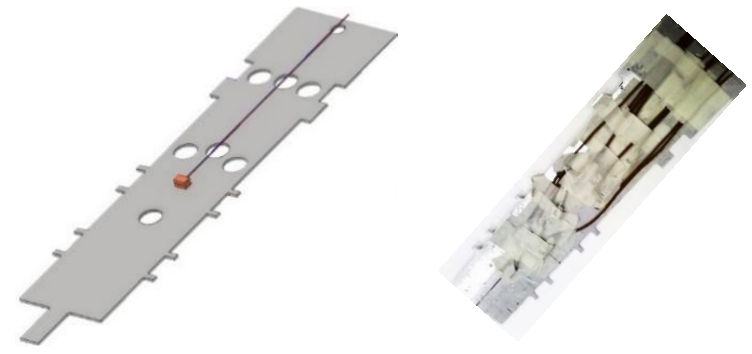

Figure 4: The model of aluminum holed plate (left) with its real picture of 9 TC on the right side.

As possible see on the right side of Figure 4, all thermocouples are very close together and due to narrow space, the insulation was not possible to be perfect. This experiment was irradiated for 5 hours by $660 \mathrm{MeV}$ protons with the total integral of particles about $4.4 \cdot 10^{15}$. Proton beam was monitored by $\mathrm{Al}$ and $\mathrm{Cu}$ activation foils with a mass $8.46 \mathrm{~g}$ and $10.96 \mathrm{~g}$. It has been found the probes react well to proton beam changes. The beam is not continuous and each small dropout was reflected by temperature fall. A similar effect is shown in the Figure 7 right.

The temperature of the sample at the position $0 \mathrm{~mm}$, where the proton beam axis is located, reacts to the proton beam change the greatest as calculation supposed, see in Figure 5 left and right. In the figure on the left side, see the temperature dependence on 5 minutes' irradiation pulses

\footnotetext{
${ }^{2}$ probe - in this article the probe is called thermocouple type T inserted in (covered by) two wafers of measured sample such as ${ }^{232} \mathrm{Th},{ }^{\text {nat }} \mathrm{U}$ or ${ }^{235} \mathrm{U}$ with mass in range of $1-2 \mathrm{~g}$
} 
before long irradiation starts. At this experiment, the contact reliability between TC and sample was tested on the probes at position $40 \mathrm{~mm}$. Finally, the result of analyzation shows, the most reliable is dry contact, in Figure 5 called 'no insulation'.

Positions at $+120 \mathrm{~mm},+80 \mathrm{~mm},+40 \mathrm{~mm}, 0 \mathrm{~mm},-40 \mathrm{~mm}$ and $-80 \mathrm{~mm}$ were monitored by similar probes with ${ }^{232} \mathrm{Th}$. It was found that insulation of probe was insufficient because the measured temperature was influenced by heat transfer of warm air up, from the positions below. Because of not bad geometry symmetry, the measured temperature should be similar at $+80 \mathrm{~mm}$ as at $-80 \mathrm{~mm}$. In fact, it was not similar too much. It the Figure 5 on the right side is shown the difference between position $+120 \mathrm{~mm}$ and $-80 \mathrm{~mm}$ with a comparison to $0 \mathrm{~mm}$. At that graph (right) the left axis represents absolute change of temperature caused by nuclear fission in probe.
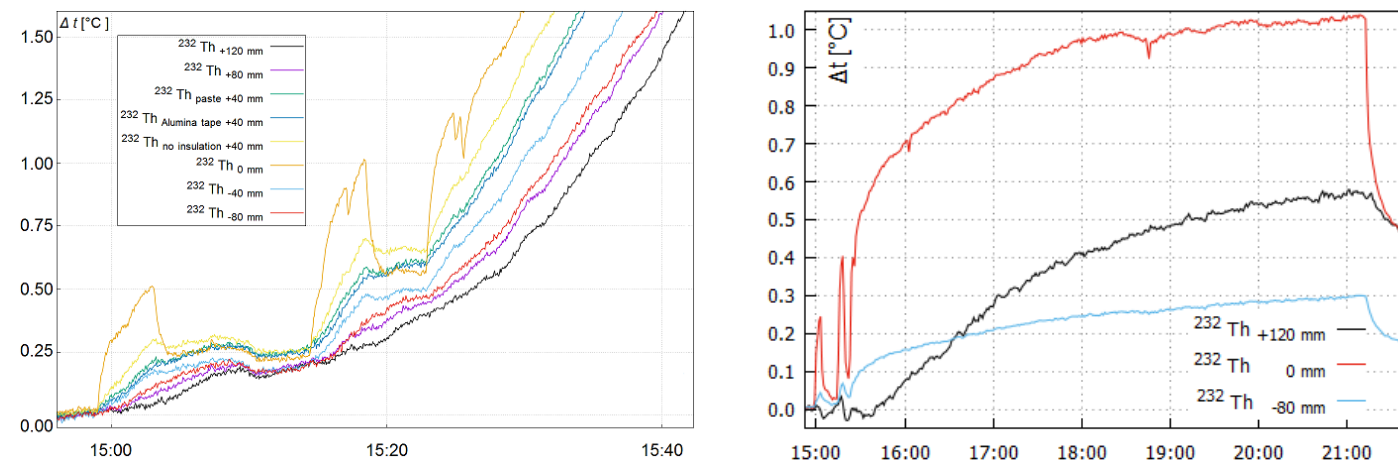

Figure 5: Graphs show the temperature changes depended on 5min pulses of irradiation for various positions on left and comparison of 3 positions of measuring during all irradiation time on the right.

Due to this experiment, new data were reached to understand heat production, transfer, and behavior inside of TA QUINTA. Because of insufficient thermal insulation, another method of measuring started to be developed.

\subsection{Experiment - March \& May 2016}

Next experiment in March was focused on confirmation and improvement of the previous result in the different position of TA QUINTA. This experiment was measured between $3^{\text {rd }}$ and $4^{\text {th }}$ section. The insulation was increased and the aluminum holder was exchanged by cardboard holder to minimalize thermal conduction transfer of holder.
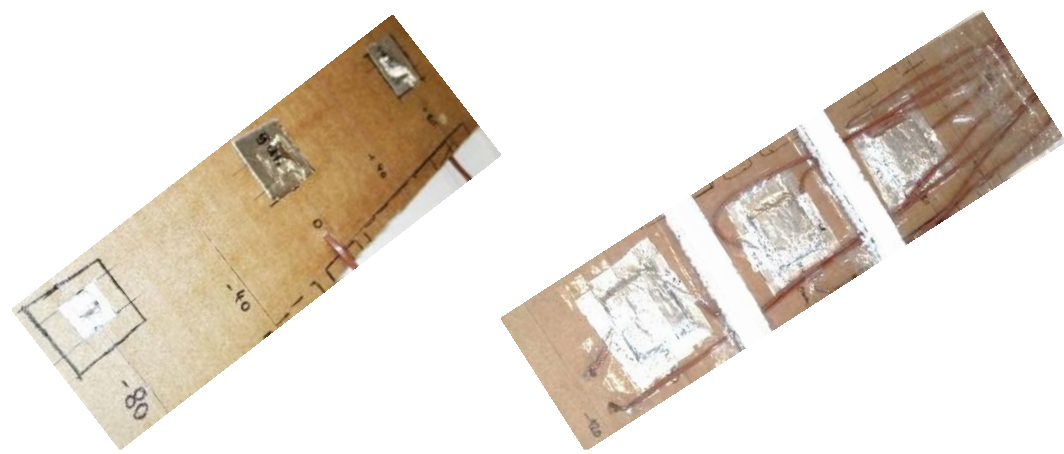

Figure 6: Picture of the insulation of probes before polystyrene cover (left) and final version (right).

Three probes with samples of ${ }^{235} \mathrm{U}$ measured the positions of $-80 \mathrm{~mm}, 0 \mathrm{~mm}$ and $+80 \mathrm{~mm}$. The insulation, which shown in Figure 6, was doubled in comparison with the previous experiment. There were 3 probes and 6 other TCs to measure online temperature outside of the probes insulation. This measurement outside of probes insulation was set to compare these two 
temperatures and evaluate the quality of insulation in these hard conditions. Insulation consisted of alumina tape to decrease transfer heat by reflection into the probe. Next insulation level was special insulating foam in combination with $5 \mathrm{~mm}$ of highly insulative polystyrene.
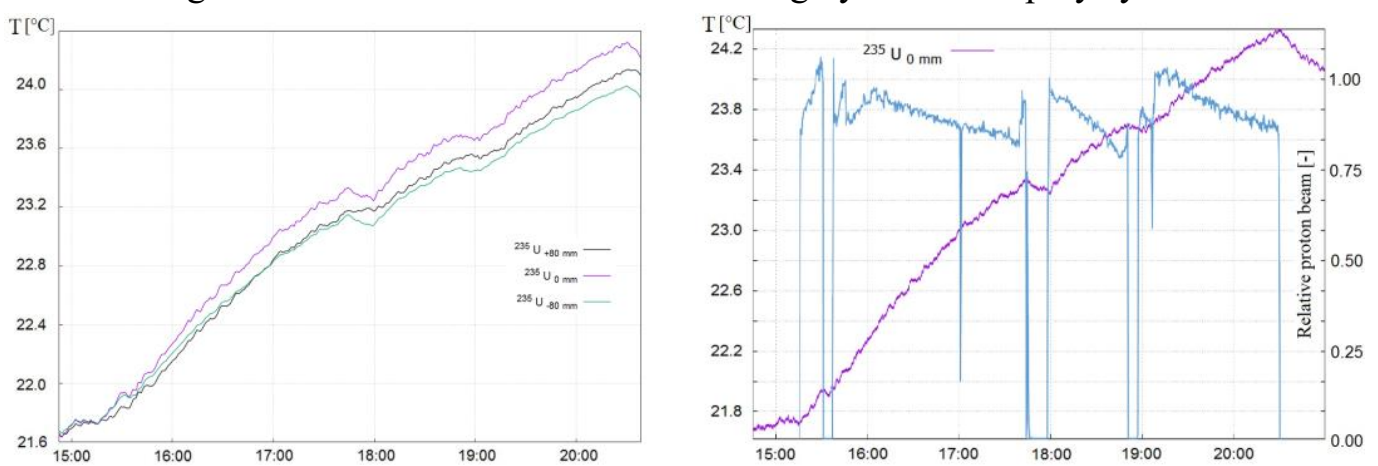

Figure 7: The left graph shows temperature increasing in probes during the time of irradiation, the right one shows the dependence of $0 \mathrm{~mm}$ located probe temperature drops on proton beam instability.

As shown in Figure 7, during 5 hours of irradiation by $660 \mathrm{MeV}$ protons of the TA QUINTA, the probes analyzed the temperature change about $2.6{ }^{\circ} \mathrm{C}$ when inserted between the 3rd and 4th section at the position $0 \mathrm{~mm}$. The graph describes this effect is shown on left of Figure 7. The most valuable result is shown on the right side. There were observed the dependence of temperature drop on the neutron field instability, or proton beam instability respective. Due to these drops, the first estimation of the heat thermal capacity of TA QUINTA was discussed. To be able to observe (or measure) and estimate the thermal capacity of the whole system, the next experiment was prepared for May 2016.

Due to a great mass of the uranium inside of the TA QUINTA, the most of the heat is produced by natural uranium cylinders from which the QUINTA consist of. All previous experiments were measured by probes with few TCs only for control measuring. In this experiment, all sections of TA QUINTA were measured at various positions to be able to compare each position in different sections. Due to that, it was found the proton beam affects the measurement the most between $1^{\text {st }}-2^{\text {nd }}$ and $2^{\text {nd }}-3^{\text {rd }}$ sections as shown in Figure 8 left measurement with the probe inside of the insulation. To compare all positions differences, see Figure 8 on the right side. Important is to compare light blue line (natural uranium probe) with yellow lines (back and front temperatures of section $2^{\text {nd }}$ ). The front section at position $0 \mathrm{~mm}$ gets warmer than backside and both were warmer than insulated probe measuring between $1^{\text {st }}$ and the $2^{\text {nd }}$ section with ${ }^{\text {nat }} U$.
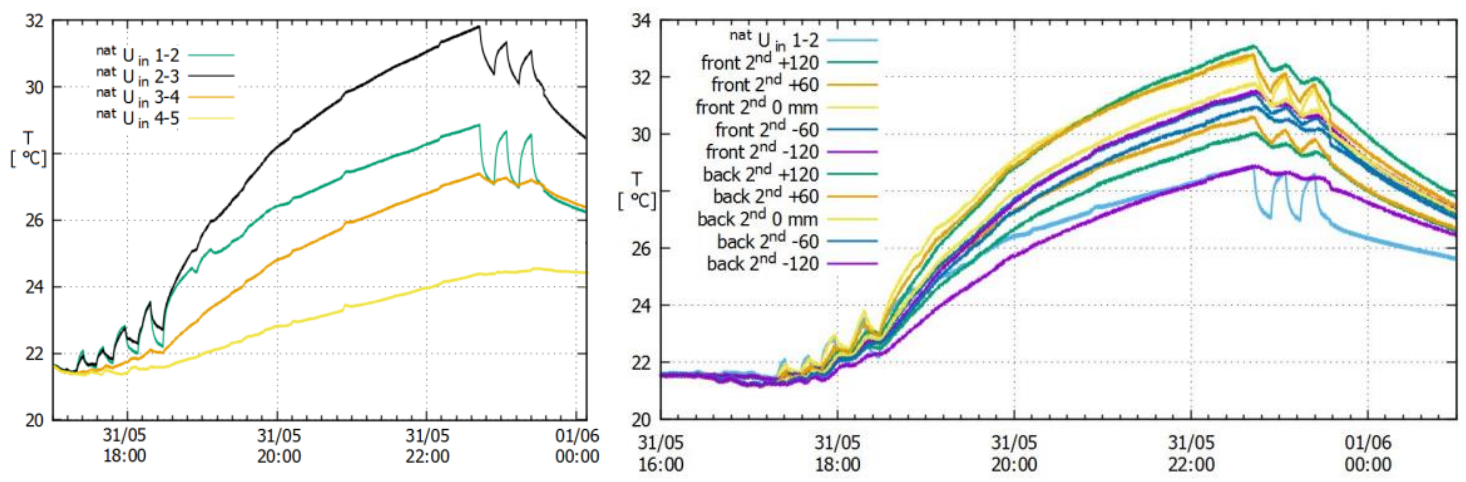

Figure 8: Comparison of all section measurement on position $0 \mathrm{~mm}$ on left and all position in from and back of $2^{\text {nd }}$ section on right. 
The reason of lots of peak with similar shape is that irradiation was not constant. The pulse irradiation at the beginning was $2 \times 5$ minutes continued by 5 min pauses. Then $2 \times 10$ min with long irradiation of $120 \mathrm{~min}$ finished by $2 \times 10$ minutes irradiation. Each $10 \mathrm{~min}$ of irradiation are related to 10 minutes' stops between irradiation pulses. Due to a shape of so-called peaks, a lot of analyzation may be made. As graphs shows, the peak shapes are similar and may be fitted to reach numeric estimation of important constants. This May experiment is going to be recalculated by advance software ANSYS ${ }^{3}$ to model heat transfer inside of TA QUINTA. Due to this the model, the numerical calculations will be improved to be more accurate. About 40 thermocouples were used for this experiment. Each thermocouple needs to be length up to $23 \mathrm{~m}$ because of strong gamma radiation and hard neutron field in the time of experiment.

\subsection{Experiment - June 2016}

June experiment brings perfect result in parallel research. The probes were measured outside of TA QUINTA to monitoring the neutron leakage, the neutrons escaping from the target. To get accurate comparable results, two probes and one TC (called TC 00) were used as shown on the left side of Figure 9. The idea was to measure the shielding of TA QUINTA where the heat from the target passes through the air from the lead. Per conditions, the temperature measured by TC 00 should be higher than temperature measured by a probe with $\mathrm{W}$ because there is no possibility to produce heat except very low gamma radiation. On the same level as $\mathrm{W}$ probe, the ${ }^{235} \mathrm{U}$ probe is located to measure heat generated by nuclear fission in this sample.

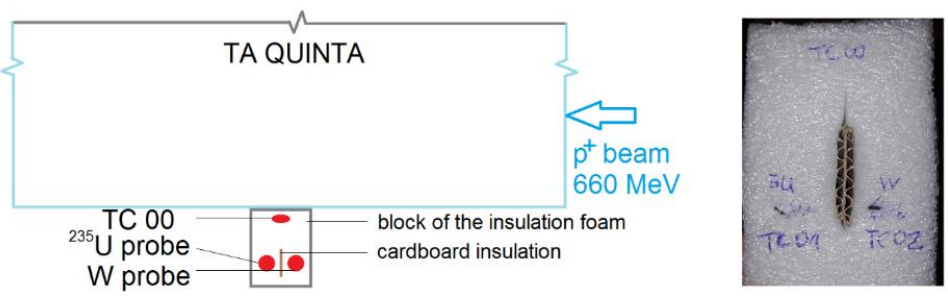

Figure 9: Setup of outside June experiment on the scheme (left) and real picture (on right).

This experiment was firstly irradiated in the pulse as the May experiment, to see how the probes react on neutron flux changes. Reached results completely confirm the theoretical assumption. Leakage neutrons were measured by comparison of temperature change between ${ }^{235} \mathrm{U}$ probe and W probe. As shown in Figure 10 the temperature of the lead is caused by heat transfer from target to the air.
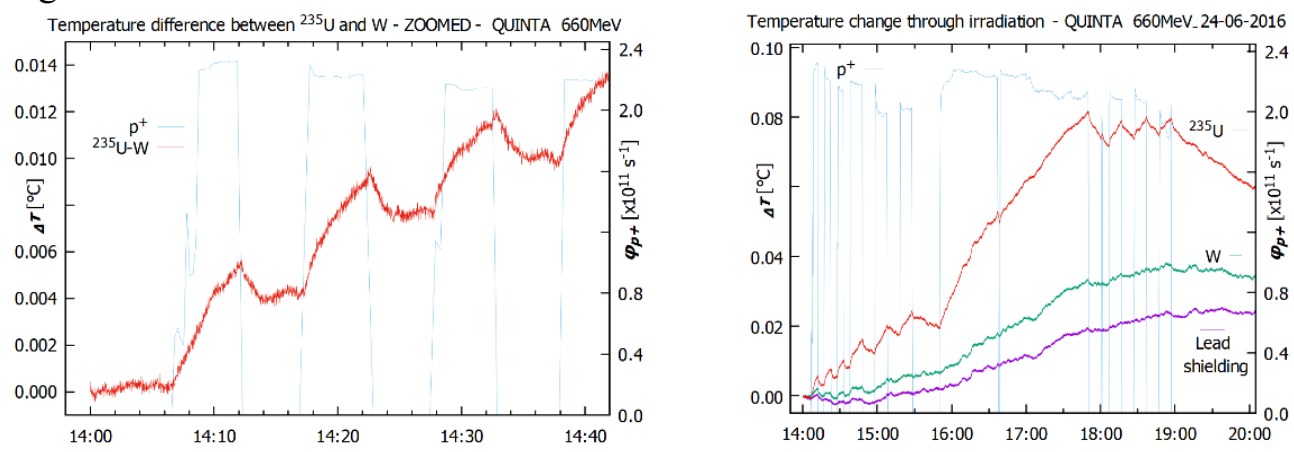

Figure 10: Zoomed shape of temperature peaks reacting on the neutron flux changes on the left and graph of TC 00 (Lead shielding), $W$ probe and ${ }^{235} U$ probe comparison on the right.

${ }^{3}$ ANSYS - Simulation software product, see http://www.ansys.com/ 


\section{Conclusion}

The neutron flux determination by temperature change inside of the spallation target QUINTA is a huge challenge because the most of the energy is generated inside of uranium cylinders. Because of narrow space between sections, the direct measurement of temperature on uranium cylinders was not possible. The indirect measurement was performed by probe consists of a thermocouple inserted into two wafers of irradiated sample covered by insulation. 4 different insulation were tested and compared, including vacuum chambers. Due to the great heat capacity of the TA QUINTA, the most of the measurement needs to be analyzed by numeric model.

Each section of TA QUINTA was monitored by TC at various positions to be able to compare real conditions with the numerical model. The most interesting result comes from the experiment measuring the neutrons leakage outside of TA QUINTA. Due to high accuracy measurement, temperature change $0.06{ }^{\circ} \mathrm{C}$ was measured between $\mathrm{W}$ probe and ${ }^{235} \mathrm{U}$ probe as shown in the graph below. Small peaks caused by pulse proton beam irradiation shows the leakage neutron dependence on spallation reaction of protons with the target.

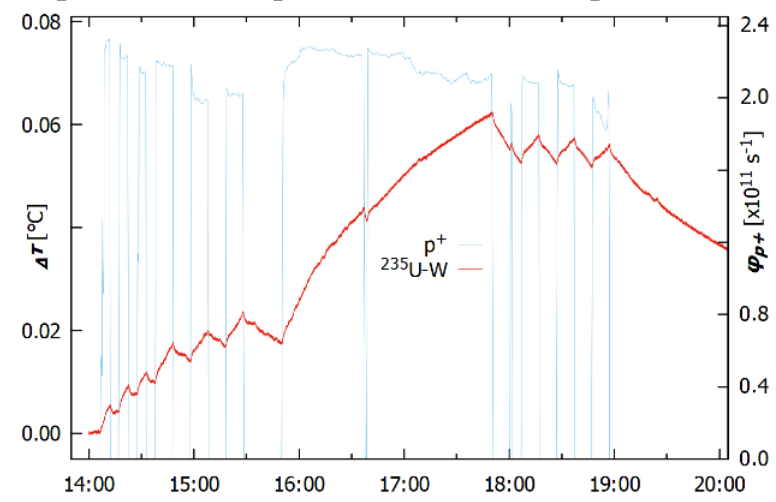

\begin{tabular}{|c|c|c|c|c|}
\hline Isotope & $Q\left[\mathbf{s}^{-1}\right]$ & $I_{\text {sample }}[-]$ & $\begin{array}{l}\text { Yield } \\
{[\%]}\end{array}$ & $\begin{array}{l}\text { Number of } \\
\text { fission }[-]\end{array}$ \\
\hline${ }^{97} \mathbf{Z r}$ & $1.94 \mathrm{E}+05$ & $2.27 \mathrm{E}+09$ & $6.00(08)$ & $3.39 \mathrm{E}+10$ \\
\hline${ }^{99}$ Mo & $2.05 \mathrm{E}+05$ & $2.40 \mathrm{E}+09$ & $5.94(08)$ & $3.74 \mathrm{E}+10$ \\
\hline${ }^{103} \mathrm{Ru}$ & $8.96 \mathrm{E}+04$ & $1.05 \mathrm{E}+09$ & $3.24(05)$ & $3.79 \mathrm{E}+10$ \\
\hline${ }^{131} \mathbf{I}$ & $1.01 \mathrm{E}+05$ & $1.18 \mathrm{E}+09$ & $3.22(05)$ & $3.81 \mathrm{E}+10$ \\
\hline${ }^{133} \mathbf{I}$ & $2.02 \mathrm{E}+05$ & $2.36 \mathrm{E}+09$ & $6.72(30)$ & $4.01 \mathrm{E}+10$ \\
\hline${ }^{140} \mathrm{Ba}$ & $1.71 \mathrm{E}+05$ & $2.00 \mathrm{E}+09$ & $5.98(06)$ & $3.38 \mathrm{E}+10$ \\
\hline${ }^{140} \mathrm{La}$ & $2.31 \mathrm{E}+05$ & $2.70 \mathrm{E}+09$ & $5.98(06)$ & $4.56 \mathrm{E}+10$ \\
\hline${ }^{143} \mathrm{Ce}$ & $1.65 \mathrm{E}+05$ & $1.93 \mathrm{E}+09$ & $5.73(08)$ & $3.86 \mathrm{E}+10$ \\
\hline \multirow[t]{2}{*}{${ }^{147} \mathrm{Nd}$} & $6.75 \mathrm{E}+04$ & $7.90 \mathrm{E}+08$ & $2.14(02)$ & $3.67 \mathrm{E}+10$ \\
\hline & & & & $3.83(7) E+10$ \\
\hline
\end{tabular}

Figure 11: Absolute temperature difference between $W$ and ${ }^{235} U$ probe at the same position on the left side and gamma-spectrometry result with heat calculation results on the right by using [4], [5].

Measured temperature shown in Figure 11 reflects heat generation inside of the measuring samples accumulated in the insulation. Heat generation measured on ${ }^{235} \mathrm{U}$ probe was caused by the neutrons leakage of TA QUINTA and reached 984(74) mJ. This result is planned to be compared with numeric models after finish necessary correction with data from upcoming May experiment. The numeric model uses results from the MCNPX calculation as the input data for accurate numeric calculation.

\section{References}

[1] L. Zavorka, Transmutation of actinides using spallation reactions, 2015, 154 p., dissertation, Czech Technical University in Prague.

[2] W. Furman, Recent results of the study of ADS with $500 \mathrm{~kg}$ natural uranium target assembly QUINTA irradiated by deuterons with energies from 1 to $8 \mathrm{GeV}$ at JINR. Proceeding of Science. 2012.

[3] J. Svoboda, Determination of the neutron flux by the temperature differences at the massive spallation uranium target QUINTA, IEEE Xplore, EPE2016.

[4] TENDL-2010, Nuclear data library, A.J. Koning and D. Rochman Release date: December 8, 2010.

[5] V. Batin et al., Temperature Measurement of the Uranium Sample Irradiated with secondary Neutrons, JINR Publishing No.5,6[97]-99. 1999, Dubna. 